\title{
ON THE RELATIVE DENSITY OF SETS OF INTEGERS ${ }^{1}$
}

\section{H. G. RICE}

In [1], U. T. Medvedev introduced the concept of relative density of sets of non-negative integers. A set $\alpha_{2}$ is at least as dense as a set $\alpha_{1}$ when there exists a general recursive function $g(x)$ such that, for every $n$, the number of elements of $\alpha_{1}$ not greater than $n$ does not exceed the number of elements of $\alpha_{2}$ not greater than $g(n)$. We give here an equivalent definition and prove a theorem which has a result of Medvedev and a result of the author as corollaries.

We assume familiarity with [3], especially $\S \S 8,16$ and 17 .

1. If $h_{i}(x)$ produces the principal sequence of $\alpha_{i}$, the number of elements of $\alpha_{i}$ not greater than $n$ is $\mu y\left[n<h_{i}(y)\right]$. So $\alpha_{2}$ is at least as dense as $\alpha_{1}$ when there exists a general recursive function $g(x)$ such that, for all $n, \mu y\left[n<h_{1}(y)\right] \leqq \mu y\left[g(n)<h_{2}(y)\right]$. We show that this is equivalent to $h_{2}(x) \leqq g\left(h_{1}(x)\right)$ for all $x$.

Suppose for all $n, \mu y\left[n<h_{1}(y)\right] \leqq \mu y\left[g(n)<h_{2}(y)\right]$. Then if for some $x, g\left(h_{1}(x)\right)<h_{2}(x)$ we have the contradiction

$$
x \geqq \mu y\left[g\left(h_{1}(x)\right)<h_{2}(y)\right] \geqq \mu y\left[h_{1}(x)<h_{1}(y)\right]=x+1 .
$$

On the other hand, suppose for a general recursive function $g(x)$, $h_{2}(x) \leqq g\left(h_{1}(x)\right)$. We may assume $g(x)$ to be monotone increasing without loss of generality. Then for any $n, h_{1}\left(\mu y\left[n<h_{1}(y)\right]-1\right) \leqq n$. Hence

$$
h_{2}\left(\mu y\left[n<h_{1}(y)\right]-1\right) \leqq g\left(h_{1}\left(\mu y\left[n<h_{1}(y)\right]-1\right)\right) \leqq g(n) .
$$

So

$$
\begin{aligned}
\mu y\left[g(n)<h_{2}(y)\right] & >\mu y\left[n<h_{1}(y)\right]-1 \\
& \geqq \mu y\left[n<h_{1}(y)\right] .
\end{aligned}
$$

We can say, then that $\alpha_{2}$ is at least as dense as $\alpha_{1}$ when there exists a general recursive function which maps $\alpha_{1}$ onto a set whose principal sequence bounds that of $\alpha_{2}$. In particular, if $\alpha \in B$, then $\alpha$ is at least as dense as $\epsilon$; this class constitutes the highest degree of density. The relation "is at least as dense as" is clearly reflexive and transitive.

We mention a fact observed by Medvedev: if $\alpha_{1} \in V-B$, then the set $\alpha_{2}=\alpha_{1}-\{a\}$, formed by removing the element $a$ from $\alpha_{1}$, is less

Received by the editors April 16, 1956.

1 The work reported in this paper was supported in part by the National Science Foundation. 
dense than $\alpha_{1}$. For when $x$ is greater than $\mu y\left[h_{1}(y)=a\right], h_{1}(x)<h_{1}(x+1)$ $=h_{2}(x)$. So $\alpha_{1}$ is at least as dense as $\alpha_{2}$. However, if $\alpha_{2}$ were at least as dense as $\alpha_{1}$, there would exist a monotone increasing general recursive function $g(x)$ such that $h_{2}(x)=h_{1}(x+1) \leqq g\left(h_{1}(x)\right) \leqq g^{x+1}\left(h_{1}(0)\right)$. Thus $\alpha_{1} \in B$, contrary to hypothesis.

2. TheOREM. $B^{*}$ is the class of automorphisms of $V$ which preserve or increase density.

Proof. We consider the automorphism $\alpha \rightarrow p(\alpha)$, and show that for all sets $\alpha, p(\alpha)$ is at least as dense as $\alpha$, if and only if there exists a general recursive function $f(x)$ such that $p(x) \leqq f(x)$ for all $x$.

First, suppose $p(x) \leqq f(x)$. Then if $\alpha_{2}=p\left(\alpha_{1}\right)$,

$$
\begin{aligned}
h_{2}(x) & \leqq \max \left[p\left(h_{1}(0)\right), p\left(h_{1}(1)\right), \cdots, p\left(h_{1}(x)\right)\right] \\
& \leqq \max \left[f(0), f(1), \cdots, f\left(h_{1}(x)\right)\right] .
\end{aligned}
$$

So $g(x)=\max [f(0), f(1), \cdots, f(x)]$ is a general recursive function for which $h_{2}(x) \leqq g\left(h_{1}(x)\right)$, and $\alpha_{2}=p\left(\alpha_{1}\right)$ is at least as dense as $\alpha_{1}$.

Conversely, suppose that $p(\alpha)$ is at least as dense as $\alpha$ for every set $\alpha$. Let $\alpha_{2}$ be the set of maximal values of $p(x)$, and $\alpha_{1}$ the set of all $y$ such that $p(y) \in \alpha_{2}$. Then $\alpha_{2}=p\left(\alpha_{1}\right)$; in fact $h_{2}(x)=p\left(h_{1}(x)\right)$ and $h_{1}(0)=0$. Now $h_{1}(x+1) \leqq h_{2}(x)+1$, for at least one of the $h_{2}(x)+2$ values of $p(y)$ for $0 \leqq y \leqq h_{2}(x)+1$ must exceed $h_{2}(x) \cdot h_{1}(x+1)$ produces the principal sequence of $\alpha_{1}-\{0\}$, so we have shown that $\alpha_{1}-\{0\}$ is at least as dense as $\alpha_{2}$. But $\alpha_{2}$ is at least as dense as $\alpha_{1}$ by hypothesis, and $\alpha_{1}$ is at least as dense as $\alpha_{1}-\{0\}$. Hence all three sets are of equal density. Since $\alpha_{1}$ and $\alpha_{1}-\{0\}$ can be of equal density only when $\alpha_{1} \in B$, we have also $\alpha_{2} \in B$ and by Theorem 22 of [3], $p(x) \leqq f(x)$ for some general recursive function $f(x)$.

Corollary [3, Theorem 23, Corollary]. B is closed under automorphisms in $B^{*}$.

Corollary. [1] Density is preserved under automorphisms in $E^{*}$.

For density is preserved by just those automorphisms which are in $B^{*}$ and whose inverses are also in $B^{*}$.

\section{REFERENCES}

1. U. T. Medvedev, On non-isomorphic recursively enumerable sets, Doklady Akademii Nauk SSSR, vol. 102 (1955) pp. 211-214 (Russian).

2. A. Mostowski, Review of [1], J. Symbolic Logic vol. 21 (1956) pp. 101-102.

3. H. G. Rice, Recursive and recursively enumerable orders, Trans. Amer. Math. Soc. vol. 83 (1956) pp. 277-300.

UNIVERSITY OF NEW HAMPSHIRE 